\title{
Periodic paralysis: what clinician needs to know?
}

\begin{abstract}
Acute flaccid paralysis is a diagnostic challenge in the emergency department. Periodic paralysis syndromes are characterized by recurrent episodes of flaccid hyporeflexic paralysis in association with potassium abnormalities. Periodic paralysis with hypokalaemia may be genetic, secondary to systemic hypokalaemia or associated with thyrotoxicosis. Genetic syndrome result from mutations in sodium (SCN4A) or calcium (CACNA1S) channels, inherited in autosomal dominant pattern. Diagnosis is established by demonstrating recurrent nature, family history and abnormal serum potassium during an episode. Thyrotoxic periodic paralysis is often sporadic but possibly has a genetic predisposition. Presence of thyrotoxicosis and hypokalaemia during an episode confirms the diagnosis. Management of acute episode is by cautiously correction of potassium abnormality. Long term therapy depends on the cause. Pathogenic mechanisms, differential diagnosis and treatment principles are discussed.
\end{abstract}

Keywords: periodic paralysis, hypokalaemia, hyperkalaemia, channelopathies, acute flaccid paralysis
Volume 6 Issue 4 - 2018

\author{
HA Dissanayake,' PACD Padmaperuma² \\ 'University Medical Unit, National Hospital of Sri Lanka, Sri \\ Lanka \\ ${ }^{2}$ Colombo North Teaching Hospital, Sri Lanka
}

Correspondence: HA Dissanayake, University Medical Unit, National Hospital Sri Lanka, Colombo I0, Sri Lanka, Tel+947l4219893, Email dissanayakeha@gmail.com

Received: June 30, 2018 | Published: July 18, 2018
Abbreviations: ABG, arterial blood gas analysis; ACTH, adrenocortical stimulating hormone; AST, aspartate transaminases; CACNA1S, calcium voltage-gated channel subunit alpha1 S; CPK, creatine phosphokinase; ECG, electrocardiogram; EMG, electromyogram; hyperKPP, hyperkalaemic periodic paralysis; hypoKPP, hypokalaemic periodic paralysis; IV, intravenous; $\mathrm{KCl}$, potassium chloride; LDH, lactate dehydrogenase; NormoKPP, Normokalaemic periodic paralysis; PP, periodic paralysis; REM, rapid eye movement sleep; RTA, renal tubular acidosis; SCN4A, sodium voltage-gated channel alpha subunit 4 ; TFT, thyroid function tests; TPP, thyrotoxic periodic paralysis

\section{Introduction}

Periodic paralysis (PP) is a rare group of clinical syndromes characterized by episodes of paralysis lasting minutes to days as a result of abnormal ion channel fluxes in skeletal muscles. Mutations of ion channels inherited in autosomal dominant pattern are the aetiology for primary PP syndromes. ${ }^{1,2}$ They are associated with changes in serum potassium and manifest in childhood to young adult age. Hypokalaemia of other aetiologies may also lead to limb weakness, and are referred to secondary periodic paralysis. Estimated global prevalence of heritable hypokalaemic and hyperkalaemic are 1:100 000 and 1:200 000 respectively. ${ }^{3}$ Men are affected more commonly than women.

Knowledge on genetic basis and therapeutic options of this group of diseases has rapidly expanded in the recent past. This review summarizes current knowledge on periodic paralysis syndromes with particular emphasis on thyrotoxic periodic paralysis.

\section{Clinicopathological syndromes}

Skeletal muscle channelopathies account for a spectrum of diseases ranging from myotonic syndromes to PP syndromes while some have overlapping features of both (Figure 1). PP syndromes are associated with mutations in sodium or calcium channels of skeletal muscles.

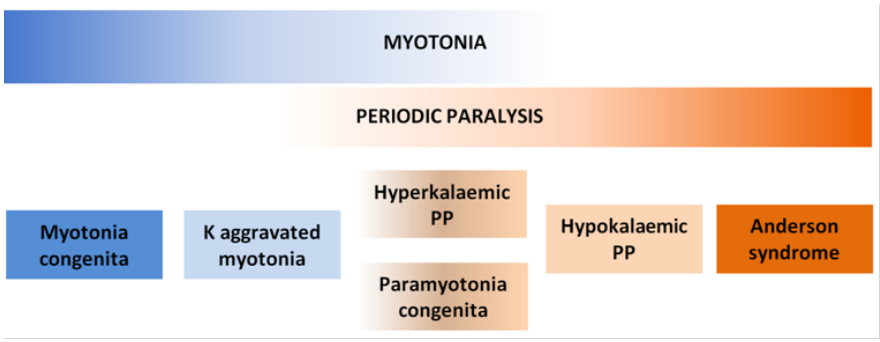

Figure I Spectrum of skeletal muscle channelopathies.

Myotonia and periodic paralysis are the cardinal manifestations of skeletal muscle channelopathies. Different mutations cause different syndromes with varying degrees of myotonia and periodic paralysis. PP, periodic paralysis; K, potassium.

\section{Hypokalaemic periodic paralysis (hypokpp)}

Periodic paralysis due to hypokalaemia may occur in three settings. 1. Inherited channelopathy causing hypokalaemia and paralysis, 2 . thyrotoxicosis precipitating hypokalaemia and paralysis, probably in genetically predisposed, and 3. hypokalaemia by other mechanisms causing paralysis (secondary hypoKPP). Two inherited channelopathies have been identified to cause hypoKPP, sodium channel mutation $(S C N 4 A)$ and calcium channel mutation (CACNA1S). Those two in general, have similar pathogenic mechanisms, clinical profiles and treatment options. However subtle differences in the phenotypes of the two types have also been recognized (see differential diagnosis). A variety of mutations in $S C N 4 A$ or $C A C N A 1 S$ responsible for disease has been identified and are inherited in autosomal dominant pattern. ${ }^{4}$

In normal health, low cytoplasmic causes hyperpolarization of skeletal muscle membrane, but when severe, leads to paradoxical depolarization, which leaves the muscle fibres insensitive to signals from neurons, thereby causing paralysis. In patients with mutated $S C N 4 A$ and CACNA1S channels, minor reduction in serum potassium is adequate to produce the paradoxical depolarization. ${ }^{4}$ Thus, the hypokalaemic paralysis develops with a minor degree of hypokalaemia 
triggered by beta adrenoceptor activation (exercise, stress etc.) or exposure to insulin, both of which activate $\mathrm{Na} / \mathrm{K}$ ATPase, causing potassium influx into cells. Muscles become depolarized and remain insensitive neural signals. Furthermore, rapid influx of potassium in to skeletal muscles traps potassium inside the cells. hypoKPP therefore represents a mechanism by which skeletal muscles have lower threshold for paradoxical depolarization with hypokalaemia and a self-perpetuating cycle of enhanced potassium influx into and trapping inside skeletal muscles (Figure 2).

Common causes for secondary hypoKPP are renal tubular acidosis (in association with connective tissue disorders), inherited renal tubulopathies (eg: Bartter syndrome, Gitelman syndrome) and dengue viral infection. ${ }^{5}$ Type 1 and type 2 renal tubular acidosis are associated with hypokalaemia due to tubular potassium loss. Similar renal potassium wasting accounts for hypokalaemia in inherited channelopathies.

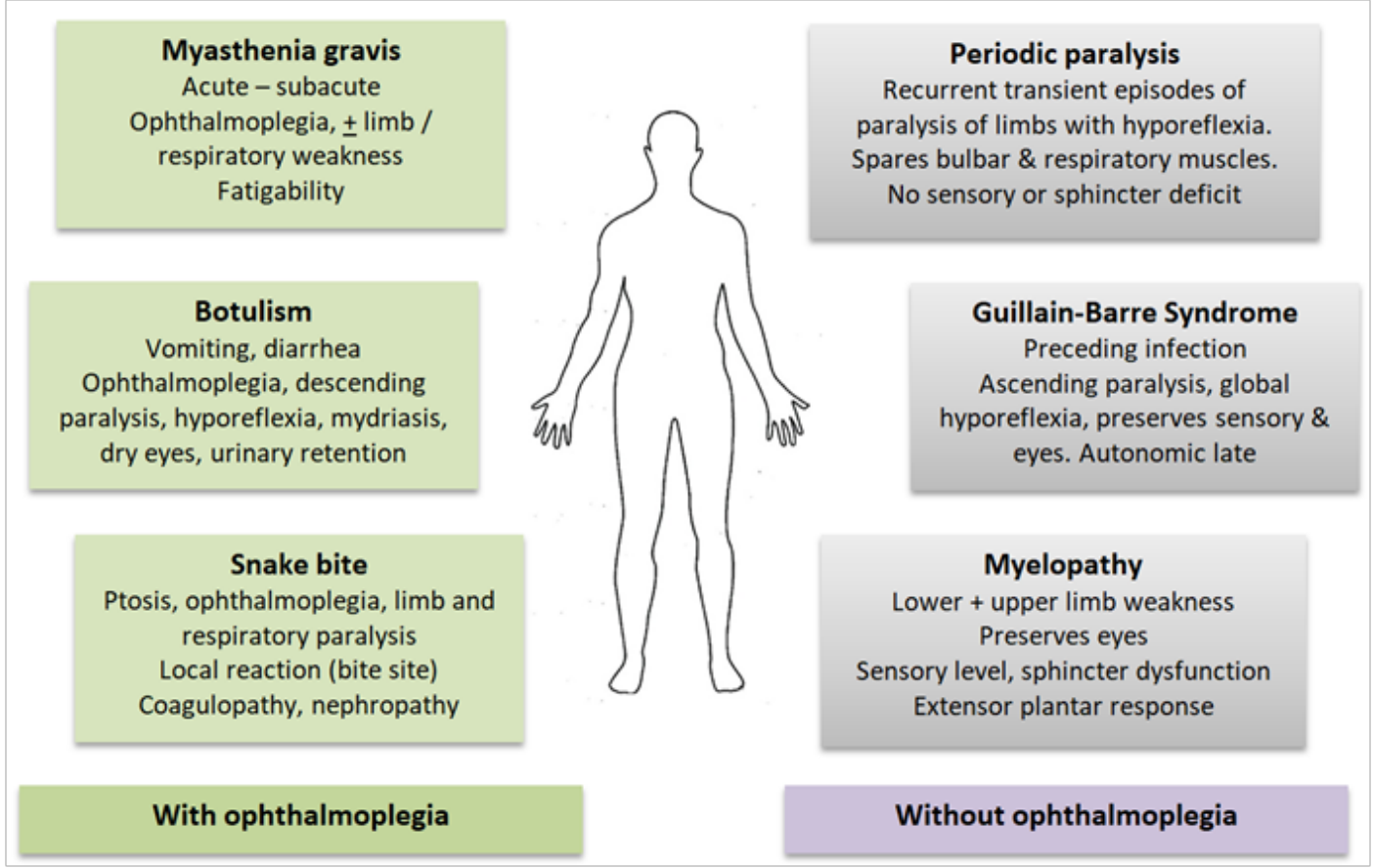

Figure 2 Clinical syndromes of acute flaccid paralysis.

Conditions in green (on the left) characteristically cause external ophthalmoplegia leading to ptosis and diplopia. These features are not seen in conditions on the right, in blue. Cardinal clinical features of differentiating value are summarized.

\section{Hyperkalaemic periodic paralysis (hyperKPP)}

Gain of function mutations in SCN4A channel lead to persistent sodium influx and sustained depolarization of skeletal muscles, which therefore become insensitive to neural stimuli. Certain SCN4A mutations cause milder degree of depolarization sufficient to cause myotonia. ${ }^{6}$ These patients are vulnerable to PP whenever sodium influx in to cells is enhanced. Sodium influx into cells is accompanied by potassium efflux, thus raising extracellular potassium.

\section{Normokalaemic periodic paralysis (normoKPP)}

Until recently, normoKPP was considered as a type of hyperKPP. It is not uncommon for the hyperKPP to present at the nadir of weakness, therefore having normal potassium level. Recently, novel mutations in SCN4A and CACNA1S genes have been identified and pathogenic mechanisms are still unclear.,

\section{Thyrotoxic periodic paralysis (TPP)}

TPP is associated with hypokalaemia. This is particularly common among people of East Asian descent, ${ }^{8}$ suggesting a genetic predisposition. Most cases are sporadic and have no family history of periodic paralysis. Possible gene responsible for this disease was identified only recently. ${ }^{9}$ Thyrotoxicosis increases cellular $\mathrm{Na} / \mathrm{K}$ ATPase activity and thereby enhancing the disease causing potential of $K C N J 2^{10-12}$ and $K C N J 18$ gene mutation. ${ }^{4}$ Yet, exact mechanism by which the above mutation causes PP is uncertain. $K C N J$ codes for a potassium influx channel and its constitutive action is postulated to be central in pathogenesis. Whether these mutations are linked to thyrotoxicosis or whether PP manifests if the individual develops thyrotoxicosis by random risk is not known. Nevertheless, thyrotoxicosis of any aetiology can precipitate TPP, suggesting the latter. Mutations in SCNAA and CANCA1S have not conclusively shown association with TPP. ${ }^{13,14}$ Interestingly, the same gene $K C N J 2$ has been implicated in the pathogenesis of Andersen-Tawil syndrome, characterized by myotonia, periodic paralysis, cardiac conduction anomalies (long QT).

Thyroxine excess also appears to increase the activity of $\mathrm{Na} / \mathrm{K}$ ATPase channel by transcriptional and post transcriptional mechanisms. ${ }^{15}$ Enhanced beta adrenoceptor sensitivity may also promote the sympathetic enhancement of $\mathrm{Na} / \mathrm{K}$ ATPase channel. ${ }^{16}$ Thyrotoxicosis is associated with hyperglycaemia which in turn can result in hyperinsulinaemia. Insulin enhances $\mathrm{Na} / \mathrm{K}$ ATPase activity 
further contributing to the pathogenesis. Periodic paralysis develops only during thyrotoxic phase, indicating the direct role of thyroxine in pathogenesis. Restoring euthyroidism therefore reliably prevents paralysis episodes.

TPP is exceedingly rare in women, despite the higher incidence of thyrotoxicosis in them. ${ }^{17}$ Androgen dependent activation of $\mathrm{Na} / \mathrm{K}$ ATPase channels that cause potassium influx into cells may at least partially explain this observation. ${ }^{18}$

\section{Clinical presentation}

Recurrent episodes of transient acute onset limb weakness define the essential feature of periodic paralysis. Characteristically, weakness affects limbs, causing flaccid, bilateral, symmetrical, predominantly proximal weakness with hyporeflexia. Eye movements, swallowing, respiration, sphincter functions, cognition and sensory system are unaffected. Duration of weakness is often limited to few hours. Uncommon manifestations include weakness of an isolated limb, duration of few minutes or few days. Unlike most other disorders of muscle weakness, respiratory paralysis is extremely rare in periodic paralysis. Although rare, hypokalaemia may cause cardiac tachyarrhythmias (atrial fibrillation, supraventricular tachycardia and ventricular tachycardia), which could potentially be fatal. Although the diseases are inherited, only one third of patients will have a family history. ${ }^{19}$ High rate of acquired mutations may at least partly account for this observation. Presence of family history, previous recurrent episodes and abnormal serum potassium during the event would strongly suggest the diagnosis.

Potassium abnormalities are the hallmark electrolyte disturbance of PP syndromes. HypoKPP is also associated with mild hypophosphatemia and hypomagnesaemia, probably secondary to sympathetic activity mediated distribution in to cells. ${ }^{20}$ Muscle enzymes (creatine phosphokinase, AST, LDH) may be elevated and rhabdomyolysis may occur in severe cases associated with hypokalaemia. (See complications below). These enzymes can also be elevated in hepatic and cardiac injury. However, in the appropriate context, they are reliable markers to detect and monitor muscle injury. If doubt exists, muscle specific isozymes can be measured. However, these are not widely available.

Thyrotoxic periodic paralysis is classically associated with hypokalaemia. Men are commonly affected, often in 20-40 year age group, in contrast to primary hypoKPP which often manifests before 20 years. ${ }^{19}$ Patient will be biochemically thyrotoxic but clinical features may vary from no symptoms to overt thyrotoxicosis. ${ }^{20}$ Obese individuals are at increased risk due to hyperinsulinaemia. Therefore, it is important to screen all patients with hypoKPP for thyrotoxicosis even in the absence of suggestive clinical features. Family history of thyroid disease may be present but period paralysis is often absent.

Factors that precipitate PP episodes are summarized in (Table 1.)Hyperinsulinaemia (following carbohydrate rich meal) and high sympathetic activity (stress, exercise) are the key factors that precipitate potassium influx in to cells causing hypokalaemia and muscle paralysis due to sustained depolarization. These factors are common to all types of PP associated with hypokalaemia. Importantly, during exercise, potassium is shifted out of the skeletal muscles which overcome the sympathetic mediated influx. Rest immediately after exercise results in influx of potassium facilitated by sympathetic overactivity which is slow to return to baseline. This explains why paralysis occurs during rest after exercise and why it is temporarily relieved by exercising again.

Many reports of TPP and hypoKPP describe predominantly nocturnal or early morning symptoms, hence the old term 'nocturnal palsy'. ${ }^{21}$ Reasons for this observation are not clear but may be due to increased sympathetic activity during rapid eye movement (REM) sleep, early morning cortisol surge, and post prandial hyperinsulinaemia after a late dinner and cold temperature at night.

Table I Periodic paralysis types

\begin{tabular}{|c|c|c|c|}
\hline & hypoKPP & Thyrotoxic PP & hyperKPP \\
\hline $\begin{array}{l}\text { Gene } \\
\text { mutation }\end{array}$ & SCNA4, CACNAIS & Uncertain.? KCNJ & SCNA4 \\
\hline Inheritance & Autosomal dominant & Acquired & $\begin{array}{l}\text { Autosomal } \\
\text { dominant }\end{array}$ \\
\hline \multirow[t]{2}{*}{ Age of onset } & Before $20-25$ years & $20-40$ years & $\begin{array}{l}\text { Before } 15 \\
\text { years }\end{array}$ \\
\hline & $\begin{array}{l}\text { High carbohydrate } \\
\text { meal } \\
\text { Rest after strenuous } \\
\text { exercise }\end{array}$ & & \\
\hline $\begin{array}{l}\text { Precipitating } \\
\text { events }\end{array}$ & $\begin{array}{l}\text { Alcohol } \\
\text { High salt diet }\end{array}$ & Same as hypoKPP & $\begin{array}{l}\text { Fasting } \\
\text { Exercise }\end{array}$ \\
\hline & Menstruation & & \\
\hline & Stress & & \\
\hline & Cold temperature & & \\
\hline & Steroids & & \\
\hline $\begin{array}{l}\text { Duration of } \\
\text { paralysis }\end{array}$ & Less than $24-48$ hours & $\begin{array}{l}\text { Less than } 24-48 \\
\text { hours }\end{array}$ & Few hours \\
\hline EMG & $\begin{array}{l}\text { Myopathy during } \\
\text { attack, normal in } \\
\text { between }\end{array}$ & $\begin{array}{l}\text { Myopathy during } \\
\text { attack, normal in } \\
\text { between }\end{array}$ & $\begin{array}{l}\text { Myopathy } \\
\text { during attack, } \\
\text { myotonia } \\
\text { in between } \\
\text { attacks }\end{array}$ \\
\hline $\begin{array}{l}\text { Acute } \\
\text { treatment }\end{array}$ & $\mathrm{KCl}$ & $\mathrm{KCl}$, propranolol & $\begin{array}{l}\text { Observe, } \\
\text { potassium } \\
\text { lowering } \\
\text { therapy }\end{array}$ \\
\hline $\begin{array}{l}\text { Long term } \\
\text { treatment }\end{array}$ & $\begin{array}{l}\text { Acetazolamide } \\
\text { Spironolactone }\end{array}$ & $\begin{array}{l}\text { Treat } \\
\text { thyrotoxicosis }\end{array}$ & $\begin{array}{l}\text { Thiazide, } \\
\text { acetazolamide }\end{array}$ \\
\hline
\end{tabular}

\section{Differential diagnosis}

Acute flaccid paralysis is not an uncommon problem in the emergency department. Differential diagnoses are summarized in Figure 2. All patients should have their serum potassium levels measured urgently. Presence of hypokalaemia should raise the diagnosis of hypoKPP. It is important to evaluate all these patients for thyrotoxicosis as well as other causes of hypokalaemia. Onset after 30 years of age, clinical features of alternative causes for hypokalaemia (diuretics, diarrhea, vomiting, connective tissue disease, kidney disease), very low serum potassium (below $2.0 \mathrm{mEq} / \mathrm{L}$ ), slow clinical recovery (more than 2 days), ${ }^{5}$ presence of hypokalaemia even when asymptomatic, presence of acidosis or alkalosis, high urinary potassium excretion (urine potassium : creatinine ratio $>2.5 \mathrm{mEq} /$ mmol) and higher creatine phosphokinase levels (mean $345 \mathrm{IU} / \mathrm{L} \mathrm{Vs}$ $1046 \mathrm{IU} / \mathrm{L})^{5}$ are indicative of alternative causes for hypokalaemia. Although hypoKPP affects men more than women, no gender predisposition was seen in secondary hypoKPP. ${ }^{5}$

Primary hypoKPP due to $S C N 4 A$ and $C A C N A 1 S$ mutations may have slightly different phenotypes. The former being less common 
and characterized by younger age at onset (childhood or adolescence), weakness with muscle pain, short duration of episodes (few hours as opposed to several hours or days in latter), less severe chronic progressive myopathy and poor response to acetazolamide and spironolactone. ${ }^{19}$

\section{Diagnostic evaluation}

Demonstration of hypokalaemia during an episode of weakness and history of recurrent episodes of paralysis and family history of similar events are often adequate for the diagnosis. Further studies are seldom required.

However, it may not be feasible with all patients, particularly if presentation is delayed, since potassium rapidly corrects by itself with recovery. Patients should be advised on early presentation and attempts should be made to assess serum electrolytes soon after symptom onset. In cases of uncertainty, genetic studies, electromyogram (EMG) and provocative testing may be useful.

Genetic studies to detect specific mutations are useful for confirmation. However, variety of mutations account for the diseases, and it is likely that not all mutations are recognized thus far. Furthermore, genetic testing is not widely available. Other tests become useful when genetic studies are unavailable or when they become negative.

EMG during an attack will show myopathic changes (low compound muscle action potential). ${ }^{22}$ These features are common to all types of PP. Nerve conduction studies are essentially normal. EMG in between episodes is particularly useful in hyperKPP where electrodiagnosis of subclinical myotonia is found in $50-75 \%$, a feature not seen with hypokalaemia associated periodic paralysis.

Provocative tests induce precipitating factors in controlled environment. This can be achieved by administration of a glucose load (increasing endogenous insulin), insulin, ACTH (permissive effect on beta adrenoceptors) or by exercise. Evidence for these methods is limited to observations from case series, but they may be useful when diagnosis is in doubt. ${ }^{19}$ Exercise provocation test is probably the safest out of provocation methods. ${ }^{23}$ However, no studies have compared sensitivity, specificity and relative safety of any of the diagnostic methods.

\section{Complications}

\section{Rhabdomyolysis}

Rhabdomyolysis is rare but well recognized and potentially life threatening complication of PP. This has been reported in several patients with hypoKPP. Exact mechanism is uncertain, but is thought to be due to ischaemia of skeletal muscles. ${ }^{24}$ Potassium causes vasodilation increasing skeletal muscle perfusion, particularly during exercise. Absence of this protective mechanism in hypoKPP leads to muscle ischaemia. Interestingly, rhabdomyolysis has not been reported with hyperKPP and is commoner in secondary hypoKPP where hypokalaemia is worse, further supporting the above hypothesis.

\section{Cardiac arrhythmia}

hypoKPP is associated with hypokalaemia related ECG changes including $U$ waves, atrial fibrillation, supraventricular tachycardia, ventricular ectopics, ventricular tachyarrhythmias and rarely bradycardia. Tachyarrhythmias are more in $\mathrm{TPP}^{20}$ probably due to effects of thyrotoxicosis itself. These are potentially fatal and need close monitoring and early treatment.

\section{Progressive myopathy}

Slowly progressive degenerative myopathy is known to affect patients with periodic paralysis. This is more common with hypoKPP than with hyperKPP, and clinically manifests in the fifth decade of life. Lower limb muscles are predominantly affected. With the onset of myopathy, episodes of paralytic attacks become infrequent. Histological characteristics are vacuoles in the centre of myofibres and absence of inflammatory cell infiltrates..$^{25,26}$ Degenerating muscles will be replaced by fatty tissue which is detectable in MRI scan. Electron microcopy will demonstrate dilatation and proliferation of T tubules. ${ }^{26}$

\section{Acute management}

Upon detection of hypokalaemia in a patient with acute flaccid paralysis, initial aim should be to prevent life threatening arrhythmias by continuous monitoring of electrocardiogram and correction of potassium. Aetiology for potassium disturbance should be explored simultaneously. Development of rhabdomyolysis should be actively observed for and preventive measures should be implemented by regular monitoring and maintaining adequate hydration. Correction of hypokalaemia should promptly reverse the paralysis, thus limiting the complications of prolonged immobilization. Sustained weakness despite correction of serum potassium should raise the suspicion of an alternative diagnosis.

Correction of potassium should be achieved with caution. In hypoKPP, correction of hypokalaemia restores cell membrane polarization, resulting in release of potassium stored in intracellular compartment causing a rebound hyperkalaemia. Therefore, it is preferable to correct serum potassium with oral (rather than intravenous) potassium supplementation and serum potassium level should be monitored up to 24 hours after correction of potassium. If intravenous administration is required, $\mathrm{KCl}$ should be infused at a slow rate not exceeding $10 \mathrm{mmol} / \mathrm{h}$. higher rates are associated with $40 \%$ risk of rebound hyperkalaemia. ${ }^{27}$ It is important not to administer glucose containing solutions or carbohydrate rich food since those trigger endogenous insulin release, further promoting potassium influx in to skeletal muscles. Physical exercise may help mild cases of hypoKPP since exercise causes release of potassium from skeletal muscles.

In patient with TPP, non-selective beta blockade with oral or intravenous propranolol may also rapidly reverse the paralysis without causing rebound hyperkalaemia according to several case reports. ${ }^{28,29}$ Its role in non thyrotoxic hypoKPP is not known.

HyperKPP patients may have normal or high serum potassium. Specific treatment is often not required during an acute attack since they are brief, resolving in few hours spontaneously. Carbohydrate food or exercise may help. However, if symptoms are severe or if cardiac arrhythmias develop, potassium lowering measures such as inhaled beta agonists (salbutamol), thiazide diuretics and intravenous calcium for cardiac membrane stabilization are useful.

normoKPP is difficult to diagnose and treat. Diagnosis can easily be overlooked due to normal serum potassium, which is uncommon with periodic paralysis. Therefore diagnosis requires exclusion of common causes. Family history and personal history of recurrent episodes should raise the suspicion. Although successful treatment 
with hydrochlorothiazide has been described (as in a case of hyperKPP) optimum treatment strategy is not established.

\section{Long term management}

Identification of triggers and their avoidance is of paramount importance. Patients with hypoKPP respond to acetozolamide. Its mechanism of action is not well understood, but appears to be independent of carbonic anhydrase inhibition. Potassium sparing diuretics (spironolactone $100 \mathrm{mg}$ daily) are the other option, either alone or in combination with acetozolamide. Potassium should be monitored for hyperkalaemia. Painful gynaecomastia is a disturbing side effect.

Regular potassium supplementation is not required. Patient can be advised to take oral potassium supplements if paralytic symptoms develop. Acetozolamide and spironolactone are effective for hypoKPP due to CACNA1S mutations. SCN4A mutant hypoKPP responds less well to above measures. In fact some of the patients with SCN4A may deteriorate with those. ${ }^{30}$ Reasons for this discrepancy are not clear. Best method of preventing TPP recurrence is by treating the thyrotoxicosis. Long term beta agonism with propranolol has also shown to prevent attacks of PP in these patients, but evidence is limited to several old case reports and series. ${ }^{31}$ Few case reports have indicated that acetazolamide and potassium sparing diuretics are thought to worsen TPP and should be avoided. ${ }^{32}$ Clearly, evidence for optimum management of TPP is lacking.

Long term treatment options for hyperKPP include acetazolamide and hydrochlorothiazide diuretics. Latter acts by maintaining low serum potassium. How acetazolamide helps in hyperKPP is not known. Patients with hyperKPP are prone to anesthesia related complications which include delayed recovery from muscle paralysis and myotonia following induction adjuncts-opiates, depolarizing muscle relaxants(causing difficulties in intubation and mechanical ventilation).

Although familial periodic paralysis syndromes are inherited in autosomal dominant pattern, role of screening family members for the disease is not clear. It may be useful if the index patient's mutation is known. Lack of wide availability, high rate of sporadic cases due to acquired mutations, variable penetrance, unavailability of an established preventive method or prophylactic therapy are the potential limitations in genetic screening for the management of periodic paralysis.

Approach to diagnosis and management of periodic paralysis are summarized in Figure 3.

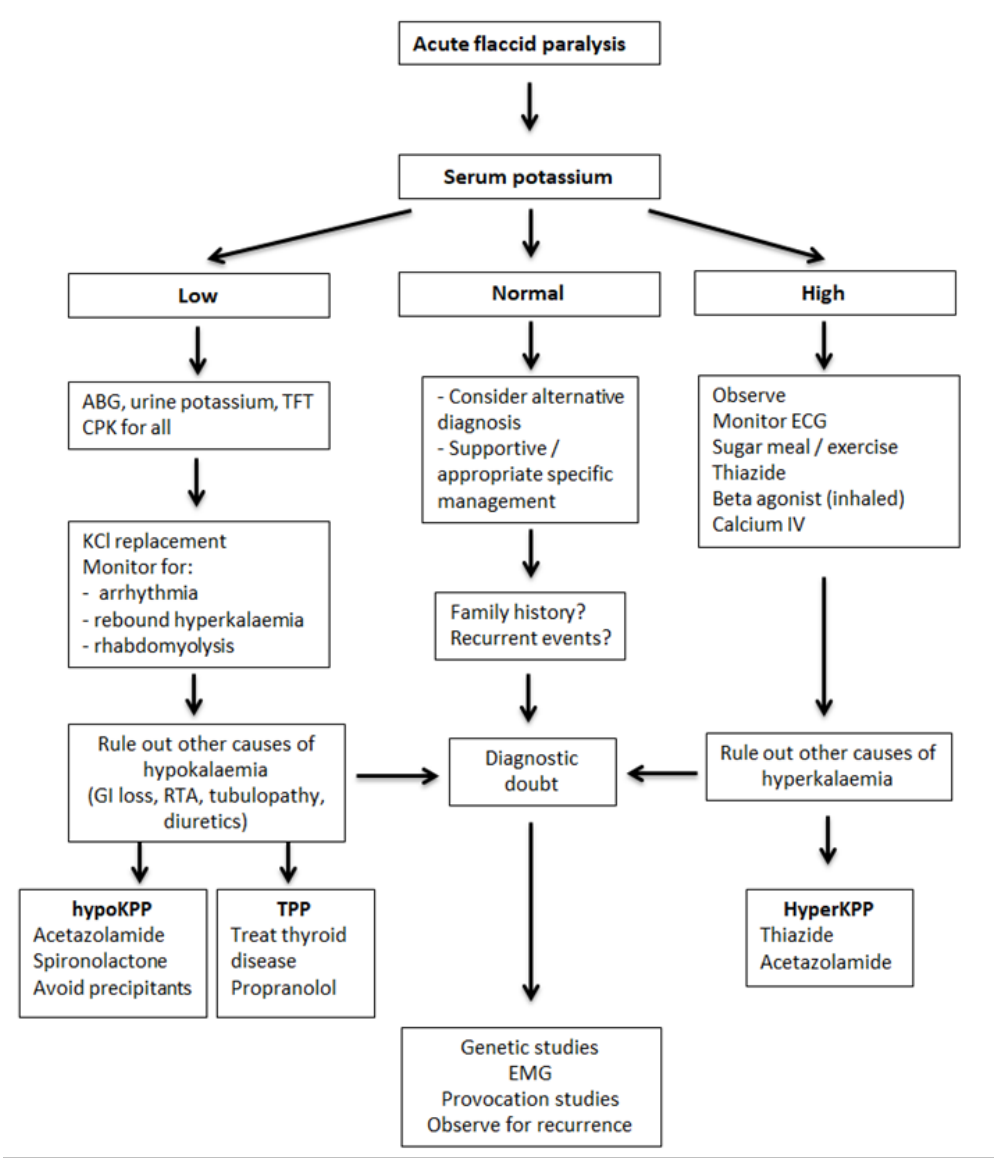

Figure 3. Diagnostic and management pathway in periodic paralysis

ABG: arterial blood gas analysis, CPK: creative phosphokinase, ECG: electrocardiogram, EMG: electromyogram, hyperKPP: hyperkalaemic periodic paralysis, hypoKPP: hypokalaemic periodic paralysis, IV: intravenous, RTA: renal tubular acidosis, TFT: thyroid function tests, TPP: thyrotoxic periodic paralysis 


\section{Summary}

Periodic paralysis syndromes are important diagnostic consideration in patients presenting with acute flaccid paralysis. Serum potassium should be tested in all, and if abnormal, should be investigated for a cause. Primary periodic paralysis syndromes are inherited and family history, onset at young age and recurrent nature along with abnormal potassium levels during episodes confirm diagnosis. Thyrotoxic periodic paralysis occurs only when the patient is thyorotoxic (at least biochemically, may be clinically silent) and is associated with hypokalaemia. All patients with hypokalemic periodic paralysis should be tested for thyrotoxicosis. Treatment of thyrotoxicosis is adequate to prevent recurrent episodes.

\section{Acknowledgements}

None.

\section{Conflicts of interest}

Authors have no conflicts of interests to declare.

\section{Refereences}

1. Cannon SC. Channelopathies of skeletal muscle excitability. Compr Physiol. 2015;5(2):761-90.

2. Fontaine B. Periodic paralysis. Adv Genet. 2008;63:3-23.

3. Jurkat-Rott K, Lerche H, Weber Y, et al. Hereditary channelopathies in neurology. Adv Exp Med Biol. 2010;686:305-34.

4. Fialho D, Griggs RC, Matthews E. Periodic paralysis. Handb Clin Neurol. 2018;148:505-520.

5. Kumar Singh A, Kumar Maurya P, Kulshreshtha D, et al. Analysis of Clinical and Metabolic Profile of Acute Neuromuscular Weakness Related to Hypokalemia. Acta Neurol Taiwan. 2017;26(3):97-105.

6. Cannon SC. Physiologic principles underlying ion channelopathies. Neurotherapeutics. 2007;4(2):174-83.

7. Fu C. Familial Normokalemic Periodic Paralysis Associated With Mutation in the SCN4A p.M1592V. Front Neurol. 2018;9:430.

8. Lin SH, Lin YF, Halperin ML. Halperin, Hypokalaemia and paralysis. QJM. 2001;94(3):133-139.

9. Lin SH, Huang CL. Mechanism of thyrotoxic periodic paralysis. J Am Soc Nephrol. 2012;23(6):985-988.

10. Cheung CL, Lau KS, Ho AY, et al. Genome-wide association study identifies a susceptibility locus for thyrotoxic periodic paralysis at 17q24.3. Nat Genet. 2012;44(9):1026-1029.

11. Jongjaroenprasert W, Phusantisampan T, Mahasirimongkol S, et al. A genome-wide association study identifies novel susceptibility genetic variation for thyrotoxic hypokalemic periodic paralysis. J Hum Genet. 2012;57(5):301-304.

12. Wang X, Chow CC, Yao X, et al. The predisposition to thyrotoxic periodic paralysis (TPP) is due to a genetic variant in the inward-rectifying potassium channel, KCNJ2. Clin Endocrinol (Oxf). 2013;80(5):770-771.

13. Tang NL, Chow CC, Ko GT, et al. The alpha (1S) subunit of the L-type calcium channel is not a predisposition gene for thyrotoxic periodic paralysis. Clin Endocrinol (Oxf). 2007;66(2):229-234.
14. Rasheed E, Seheult J, Gibney J, et al. ANNALS EXPRESS: Does thyrotoxic periodic paralysis have a genetic predisposition? A case report. Ann Clin Biochem. 2018: 4563218785395.

15. Chaudhury S, Ismail-Beigi F, Gick GG, et al. Effect of thyroid hormone on the abundance of $\mathrm{Na}, \mathrm{K}$-adenosine triphosphatase alpha-subunit messenger ribonucleic acid. Mol Endocrinol. 1987;1(1):83-89.

16. Layzer RB. Periodic paralysis and the sodium-potassium pump. Ann Neurol. 1982;11(6):547-552.

17. Manoukian MA, Foote JA, Crapo LM. Clinical and metabolic features of thyrotoxic periodic paralysis in 24 episodes. Arch Intern Med. 1999;159(6):601-606.

18. Falhammar H, Thoren M, Calissendorff J. Thyrotoxic periodic paralysis: clinical and molecular aspects. Endocrine. 2012;43(2):274-284.

19. Statland JM, Fontaine B, Hanna MG, et al. Review of the Diagnosis and Treatment of Periodic Paralysis. Muscle Nerve. 2017;57(4):522-530.

20. Kung AW. Clinical review: Thyrotoxic periodic paralysis: a diagnostic challenge. J Clin Endocrinol Metab. 2006;91(7):2490-2495.

21. Venance SL, Cannon SC, Fialho D, et al. The primary periodic paralyses: diagnosis, pathogenesis and treatment. Brain. 2006;129(Pt 1):8-17.

22. Tengan $\mathrm{CH}$, Antunes AC, Gabbai AA, et al. The exercise test as a monitor of disease status in hypokalaemic periodic paralysis. J Neurol Neurosurg Psychiatry. 2004;75(3):497-499.

23. Gyu Sik Kim, Youn Min Oh, Seung Min Kim, et al. Electrophysiological Changes by Exercise and Cold Provocation Test in a Patient with Hyperkalemic Periodic Paralysis. Journal of the Korean Neurological Association. 2000;18(5):665-668.

24. Gennari FJ. Hypokalemia. N Engl J Med. 1998;339(7):451-458.

25. Basali D, Prayson RA. Prayson, Episodic weakness and vacuolar myopathy in hypokalemic periodic paralysis. $J$ Clin Neurosci. 2015;22(11):1846-1847.

26. Jurkat-Rott K, Lehmann-Horn F. Paroxysmal muscle weakness: the familial periodic paralyses. J Neurol. 2006;253(11):1391-1398.

27. Lu KC, Hsu YJ, Chiu JS, et al. Effects of potassium supplementation on the recovery of thyrotoxic periodic paralysis. Am J Emerg Med. 2004;22(7):544-547.

28. Tassone H, Moulin A, Henderson SO. The pitfalls of potassium replacement in thyrotoxic periodic paralysis: a case report and review of the literature. J Emerg Med. 2004;26(2):157-161.

29. Lin SH, Lin YF. Propranolol rapidly reverses paralysis, hypokalemia, and hypophosphatemia in thyrotoxic periodic paralysis. Am J Kidney Dis. 2001;37(3):620-623.

30. Matthews E, Portaro S, Ke Q, et al. Acetazolamide efficacy in hypokalemic periodic paralysis and the predictive role of genotype. Neurology. 2011;77(22):1960-1964.

31. Yeung RT, Tse TF. Thyrotoxic periodic paralysis. Effect of propranolol. Am J Med. 1974;57(4):584-590.

32. Chen YC, Fang JT, Chang CT, et al. Thyrotoxic periodic paralysis in a patient abusing thyroxine for weight reduction. Ren Fail. 2001;23(1):139-142. 